\title{
PAIRS OF ADDITIVE EQUATIONS III: QUINTIC EQUATIONS
}

\author{
by R. J. COOK
}

(Received 15th March 1982)

\section{Introduction}

We consider $R$ simultaneous equations of additive type

$$
f_{i}(\mathbf{x})=a_{i 1} x_{1}^{k}+\cdots+a_{i N} x_{N}^{k}=0, \quad 1 \leqq i \leqq R,
$$

where the coefficients $a_{i j}$ are integers. Artin's conjecture, for additive forms, is that the equations (1) have a non-trivial solution in integers $x_{1}, \ldots, x_{N}$ provided that they have a non-trivial real solution, which is clearly satisfied when $k$ is odd, and

$$
N \geqq R k^{2}+1 \text {. }
$$

When $R=1$ this conjecture was proved by H. Davenport and D. J. Lewis [8] except when $7 \leqq k \leqq 17$, and $\mathrm{R}$. C. Vaughan [13] proved the result when $11 \leqq k \leqq 17$. When $R=2$ the conjecture has been proved when $k=3$, see Davenport and Lewis [9], Cook [4] and Vaughan [14], and for odd $k \geqq 19$, see Cook [5]. In the case of two quadratic equations 9 variables are sufficient provided that every form in the pencil is an indefinite quadratic form containing at least five variables explicitly, see Cook [2]. Davenport and Lewis [10] have shown that when $k$ is odd $2 k^{2}+1$ variables are sufficient to ensure the solubility of two additive equations in every $p$-adic field.

Theorem 1. Two additive equations of degree 5

$$
\begin{aligned}
& a_{1} x_{1}^{5}+\cdots+a_{N} x_{N}^{5}=0 \\
& b_{1} x_{1}^{5}+\cdots+b_{N} x_{N}^{5}=0
\end{aligned}
$$

with integer coefficients and $N \geqq 51$ variables $x_{1}, \ldots, x_{N}$ have a non-trivial solution in integers.

The proof of Theorem 1 depends on an analogue of Theorem 2 of Davenport [7] concerning admissible exponents. We obtain this, Theorem 3 , in a form suitable for an application to $R$ additive equations although at present there is inadequate information about the $p$-adic solutions of $R \geqq 3$ quintic equations. When $R$ is fixed and $k>k_{0}(\varepsilon, R)$ is 
a sufficiently large odd integer we have recently shown [6] that for any $\varepsilon>0$

$$
N \geqq \frac{(2+\varepsilon)}{\log 2} R^{2} k \log k
$$

variables are sufficient to ensure that the equations (1) have a non-trivial solution in every $p$-adic field.

Theorem 2. Let $\varepsilon>0, R \geqq 2$ and let $k>k_{0}(\varepsilon, R)$ be an odd integer. If $N$ satisfies (4) then the equations (1) have a non-trivial solution in integers $x_{1}, \ldots, x_{N}$.

For large odd $k$ this improves on Theorem 1 of Davenport and Lewis [11] which states that

$$
N \geqq 9 R^{2} k \log 3 R k
$$

variables are sufficient when $k$ is odd.

\section{Admissible Exponents}

The $s$ real numbers $\lambda_{1}, \ldots, \lambda_{s}$ satisfying

$$
\lambda_{1} \geqq \lambda_{2} \geqq \cdots \geqq \lambda_{s}>0
$$

will be called admissible exponents (for $R$ additive equations of degree $k$ ) if for any $R \times s R$ integer matrix $A$, whose columns form $s$ consecutive non-singular $R \times R$ matrices, the number of solutions of

$$
A X=A Y
$$

where

$$
X=\left[x_{1}^{k}, x_{2}^{k}, \ldots, x_{s R}^{k}\right]^{T}, Y=\left[y_{1}^{k}, y_{2}^{k}, \ldots, y_{s R}^{k}\right]^{T}
$$

are integer vectors satisfying

$$
c_{j} P^{\lambda_{v}}<x_{j}, y_{j}<C_{j} P^{\lambda_{v}}
$$

with $v=1+[(j-1) / R]$ and $0<c_{j}<C_{j}$, is

$$
O\left(P^{R\left(\lambda_{1}+\lambda_{2}+\cdots+\lambda_{s}\right)+\varepsilon}\right) \text { as } P \rightarrow \infty
$$

for any $\varepsilon>0$.

If $\lambda_{1}, \ldots, \lambda_{s}$ are admissible exponents and $\theta>0$ then $\theta \lambda_{1}, \ldots, \theta \lambda_{s}$ are also admissible exponents and also, for any $r \leqq R \lambda_{1}, \ldots, \lambda_{s}$ are admissible exponents for $r$ additive 
equations of degree $k$. Our next result is the analogue for $R$ equations of Theorem 2 of Davenport [7], the case $R=2, s=k=3$ will give Lemma 19 of Davenport and Lewis [9].

Theorem 3. Let $\lambda_{1}, \ldots, \lambda_{s}$ be admissible exponents for $R$ additive equations of degree $k$. Let

$$
\sigma=\lambda_{1}+\cdots+\lambda_{s} \text { and } \delta=k \lambda_{1}-(k-1)
$$

If $\delta>0$ then $1, \lambda_{1}, \ldots, \lambda_{s}$ are also admissible exponents provided that there exists an integer $\ell$ satisfying

$$
\begin{gathered}
1 \leqq \ell \leqq k-2, \\
2^{\ell} \delta \leqq 1, \\
\left(2^{\ell}-1\right) \delta+\sigma \leqq \ell+1 .
\end{gathered}
$$

Proof. Since the first $R$ columns of the matrix of coefficients are in general position we can take suitable linear combinations of the equations to diagonalise the first $R$ columns. It is then sufficient to prove that the number of solutions of a set of $R$ equations

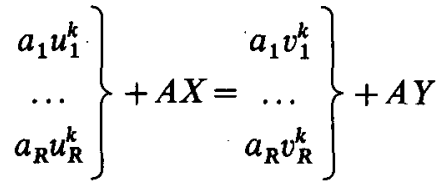

where $a_{1} \ldots a_{R} \neq 0, A, X$ and $Y$ satisfy the conditions stated in the definition, and

$$
c P<u_{i}, v_{i}<C P \text { for } i=1, \ldots, R \text {, }
$$

with $0<c<C$, is

$$
O\left(P^{R(1+\sigma)+\varepsilon}\right) \text { as } P \rightarrow \infty
$$

for any $\varepsilon>0$.

Let $N(j), 0 \leqq j \leqq R$, denote the number of solutions of (15) subject to the extra condition that $u_{i} \neq v_{i}$ for exactly $j$ values of the suffix $i$. Since $\lambda_{1}, \ldots, \lambda_{s}$ are admissible exponents

$$
N(0) \ll P^{R(1+\sigma)+\varepsilon} \quad \text { as } P \rightarrow \infty .
$$

For $j>0$ it is sufficient to prove that the number $N^{\prime}(j)$ of solutions of a system

$$
\left.\left.\begin{array}{c}
a_{1} u_{1}^{k} \\
\ldots \\
a_{j} u_{j}^{k}
\end{array}\right\}+A X=\begin{array}{r}
a_{1} v_{1}^{k} \\
\ldots \\
a_{j} v_{j}^{k}
\end{array}\right\}+A Y
$$


in variables $u_{1}, \ldots, u_{j}, v_{1}, \ldots, v_{j}, X$ and $Y$, subject also to $u_{i}>v_{i}$, is

$$
\ll P^{R(1+\sigma)-(R-j)+\varepsilon}=P^{R \sigma+j+\varepsilon} \quad \text { as } P \rightarrow \infty
$$

Let $\Delta_{t}(f(x))=f(x+t)-f(x), u_{i}=v_{i}+t(i)$ for $i=1, \ldots, j$ and then write $u_{i}$ in place of $v_{i}$. The equations (19) become

$$
\left.\begin{array}{c}
a_{1} \Delta_{t(1)}\left(u_{1}^{k}\right) \\
\cdots \\
a_{j} \Delta_{t(j)}\left(u_{j}^{k}\right)
\end{array}\right\}+A X=A Y
$$

so that

$$
0<t(i) \ll P^{\delta} \quad \text { for } \quad i=1, \ldots, j \text {. }
$$

Let $\mathbf{m}=\left[m_{1}, \ldots, m_{R}\right]^{T}$ be an integer vector and let $r(\mathbf{m})$ and $r(\mathbf{t}, \mathbf{m})$ denote the number of representations of $\mathbf{m}$ by the right and left sides of (21) respectively, with the variables lying in the specified ranges. Then

$$
\begin{aligned}
N^{\prime}(j) & \leqq \sum_{\mathbf{t}} \sum_{\mathbf{m}} r(\mathbf{m}) r(\mathbf{t}, \mathbf{m}) \\
& \leqq\left\{\sum_{\mathbf{t}} \sum_{\mathbf{m}} r^{2}(\mathbf{m})\right\}^{1 / 2}\left\{\sum_{\mathbf{t}} \sum_{\mathbf{m}} r^{2}(\mathbf{t}, \mathbf{m})\right\}^{1 / 2} \\
& \ll\left(P^{j \delta+R \sigma+\varepsilon}\right)^{1 / 2} M_{1}^{1 / 2}(j),
\end{aligned}
$$

say. It is now sufficient to prove that for any $\varepsilon>0$

$$
M_{1}=M_{1}(j) \ll P^{R \sigma+j(2-\delta)+\varepsilon} \quad \text { as } P \rightarrow \infty .
$$

For $1 \leqq i \leqq j$ and $1 \leqq h \leqq \ell$ let $u(i, h)=\left(u_{i 1}, \ldots, u_{i h-1}\right)$ be an integer vector satisfying

$$
0<u_{i n} \ll P \quad \text { for } \quad 1 \leqq i \leqq j, 1 \leqq n \leqq h-1 .
$$

We write

$$
\Delta_{t(i), u(i, h)} \text { for } \Delta_{t(i), u_{i 1}, \ldots, u_{i h-1}} .
$$

Let $M_{h}=M_{h}(j)$ denote the number of solutions of

$$
\left.\left.\begin{array}{c}
a_{1} \Delta_{t(1) u(1, h)}\left(u_{1}^{k}\right) \\
\ldots \\
a_{j} \Delta_{t(j) u(j, h)}\left(u_{j}^{k}\right)
\end{array}\right\}+A X=\begin{array}{c}
a_{1} \Delta_{t(1) u(1, h)}\left(v_{1}^{k}\right) \\
\ldots \\
a_{j} \Delta_{t(j) u(j, h)}\left(v_{j}^{k}\right)
\end{array}\right\}+A Y
$$

in all these variables, lying in the specified ranges. 
Let

$$
\alpha(h)=-\left(2^{h}-1\right) \delta+h+1,
$$

then from (24) it is now sufficient to prove that for $1 \leqq h \leqq \ell$

$$
M_{h}(j) \ll P^{R \sigma+j \alpha(h)+\varepsilon} \quad \text { as } \quad P \rightarrow \infty .
$$

We use induction on $j$, when $j=0(28)$ is true because $\lambda_{1}, \ldots, \lambda_{s}$ are admissible exponents. The contribution to $M_{h}(j)$ coming from those sets of variables such that $u_{i}=v_{i}$ for exactly $s>0$ of the suffixes $1,2, \ldots, j$ is

$$
\begin{aligned}
& \ll P^{s} M_{h}(j-s) \ll P^{R \sigma+(j-s) \alpha(h)+s+\varepsilon} \\
& \ll P^{\sigma+j \alpha(h)+s((2 h-1) \delta-h\}+\varepsilon} \\
& \ll P^{R \sigma+j \alpha(h)+\varepsilon}
\end{aligned}
$$

since

$$
2^{h} \delta \leqq 2^{\ell} \delta \leqq 1 \leqq h+\delta .
$$

Now we may suppose that for $1 \leqq i \leqq j$

$$
u_{i}=v_{i}+u_{i h} \quad \text { where } \quad 0<u_{i h} \ll P .
$$

Writing $v_{i}$ in place of $u_{i}$ the equations (26) become

$$
\left.\begin{array}{c}
a_{1} \Delta_{t(1) u(1, h+1)}\left(u_{1}^{k}\right) \\
\ldots \\
a_{j} \Delta_{t(j) u(j, h+1)}\left(u_{j}^{k}\right)
\end{array}\right\}+A X=A Y .
$$

Let $r(\mathbf{t}, \mathbf{u}, \mathbf{m})$ denote the number of representations of the integer vector $\mathbf{m}$ by the left side of (31) with the variables lying in the specified ranges, then the number of solutions of (31) that are now under consideration is at most

$$
\begin{aligned}
\sum_{t, \mathbf{u}, \mathbf{m}} r(\mathbf{m}) r(\mathbf{t}, \mathbf{u}, \mathbf{m}) & \leqq\left\{\sum_{h, 0} \sum_{\mathbf{m}} r^{2}(\mathbf{m})\right\}^{1 / 2}\left\{\sum_{\mathbf{t}, \mathbf{w}, \mathbf{m}} r^{2}(\mathbf{t}, \mathbf{u}, \mathbf{m})\right\}^{1 / 2} \\
& \ll\left\{P^{j(\delta+h)+R \sigma+\varepsilon}\right\}^{1 / 2} M_{h+1}^{1 / 2}(j) .
\end{aligned}
$$

Thus for $1 \leqq h \leqq \ell-1$

$$
M_{h}(j) \ll P^{R \sigma+j \alpha(h)+\varepsilon}+P^{(R \sigma+j(\delta+h)+\varepsilon) / 2} M_{h+1}^{1 / 2}(j) .
$$


We now estimate $M_{\ell}(j)$. If $u_{i}=v_{i}$ for some $i \leqq j$ then the inductive hypothesis shows that this contribution to $M_{\ell}(j)$ is

$$
\ll P^{R \sigma+j \alpha(\ell)+\varepsilon}
$$

If $u_{i} \neq v_{i}$ for $i=1, \ldots, j$ then we consider any $X, Y$ satisfying the last $R-j$ equations of $A X=A Y$. Since $\lambda_{1}, \ldots, \lambda_{s}$ are admissible exponents for $R-j$ equations the number of solutions involved, subject to (9), is

$$
\ll P^{2 R \sigma-(R-j) \sigma+\varepsilon}=P^{R \sigma+j \sigma+\varepsilon} .
$$

For each such $X, Y$ the remaining variables are determined to within $O\left(P^{\varepsilon}\right)$ possibilities, since for $1 \leqq i \leqq j, t(i), u_{i n}$ and $u_{i}-v_{i}$ are divisors of a fixed non-zero number that is $O\left(P^{k}\right)$ and $\Delta_{t, u}\left(u_{i}^{k}\right)$ is of degree at least 2 in $u_{i}$. Thus

$$
\begin{aligned}
M_{\ell}(j) & \ll P^{R \sigma+j \alpha(\ell)+\varepsilon}+P^{R \sigma+j \sigma+\varepsilon} \\
& \ll P^{R \sigma+j \alpha(\ell)+\varepsilon}
\end{aligned}
$$

since $\sigma \leqq \alpha(\ell)$, from (14).

Now

$$
\alpha(h)=\frac{1}{2}(\delta+h)+\frac{1}{2} \alpha(h+1)
$$

so from (33) we have

$$
M_{h}(j) \ll P^{R \sigma+j \alpha(h)+\varepsilon} \text { for } 1 \leqq h \leqq \ell
$$

which completes the proof.

\section{A calculation}

If $\lambda_{1}, \ldots, \lambda_{s}$ are admissible exponents with

$$
\lambda_{1}=1, \sigma=\sigma(s)=\lambda_{1}+\cdots+\lambda_{s}
$$

then for any $\theta>0, \theta \lambda_{1}, \ldots, \theta \lambda_{s}$ are also admissible exponents. We apply Theorem 3 with $k=5$ and see that if

$$
\theta=\theta(s)>4 / 5
$$

and either

$$
\begin{gathered}
\ell=1, \theta=\min (9 / 10,6 /(5+\sigma)), \text { or } \\
\ell=2, \theta=\min (17 / 20,15 /(15+\sigma)), \text { or }
\end{gathered}
$$




$$
\ell=3, \theta=\min (33 / 40,32 /(35+\sigma))
$$

then $1, \theta \lambda_{1}, \ldots, \theta \lambda_{s}$ are admissible exponents with

$$
\sigma(s+1)=1+\theta \sigma(s) .
$$

From our version of Hua's Lemma [3] we see that 1,1 are admissible exponents. Iterating from this result we obtain the numerical results in Table 1, the essential feature of these results is that $\sigma=\sigma(10)$ satisfies

$$
2 \sigma+1 / 2=10.025448 \ldots>10 .
$$

Table 1

\begin{tabular}{ccccc}
\hline$s$ & $\sigma(s)$ & $\ell$ & $\theta(s)$ & $\sigma(s+1)$ \\
\hline 2 & 2 & 1 & $6 / 7$ & $19 / 7$ \\
3 & $19 / 7$ & 2 & 0.846774 & 3.298387 \\
4 & 3.298387 & 3 & $33 / 40$ & 3.721169 \\
5 & 3.721169 & 3 & $33 / 40$ & 4.069964 \\
6 & 4.069964 & 3 & 0.819043 & 4.333478 \\
7 & 4.333478 & 3 & 0.813556 & 4.525528 \\
8 & 4.525528 & 3 & 0.809603 & 4.663883 \\
9 & 4.663883 & 3 & 0.806779 & 4.762724 \\
\hline
\end{tabular}

\section{Allocation of variables}

In proving Theorem 1 we can obviously make the additional assumptions that $N=51$ and that each column of coefficients contains at least one non-zero entry. Davenport and Lewis [8] have pointed out that a single quintic equation

$$
a_{1} x_{1}^{5}+\cdots+a_{n} x_{n}^{5}=0
$$

has a non-trivial solution in integers provided that $n \geqq 23$. Suppose first that 22 or more of the ratios $a_{i} / b_{i}$ occurring in (3) are equal, then the equations (3) are equivalent to a pair

$$
\left.\begin{array}{r}
a_{1}^{\prime} x_{1}^{5}+\cdots+a_{22}^{\prime} x_{22}^{5}+a_{23}^{\prime} x_{23}^{5}+\cdots+a_{N}^{\prime} x_{N}^{5}=0 \\
b_{23}^{\prime} x_{23}^{5}+\cdots+b_{N}^{\prime} x_{N}^{5}=0
\end{array}\right\}
$$

after renumbering the variables. Let $\xi_{23}, \ldots, \xi_{N}$ be a non-trivial integer solution of the second equation, and then solve the single equation

$$
a_{1}^{\prime} x_{1}^{5}+\cdots+a_{22}^{\prime} x_{22}^{5}+\left(a_{23}^{\prime} \xi_{23}^{5}+\cdots+a_{N}^{\prime} \xi_{N}^{5}\right) t^{5}=0
$$

to give a solution of the equations (47). Thus we may assume for the rest of the proof of Theorem 1 that no ratio $a_{i} / b_{i}$ occurs more than 21 times. In order to allocate the variables suitably we need a combinatorial lemma. 
Lemma 1. The 51 ratios $a_{i} / b_{i}$, with no ratio occurring more than 21 times, can be partitioned into two sets $\mathscr{S}$ and $\mathscr{T}$ such that

$$
|\mathscr{S}|=15,|\mathscr{T}|=36
$$

no ratio occurs more than 5 times in $\mathscr{S}$ and no ratio occurs more than 16 times in $\mathscr{T}$.

Proof. If no ratio occurs more than 5 times the result is trivial. Otherwise we begin with the most common ratio $a_{i} / b_{i}$, put 5 of the terms into $\mathscr{S}$ and the remaining ratios of that value (at most 16 ) into $\mathscr{T}$. We repeat this for the second most common ratio $a_{i} / b_{i}$, then the third and so on until we reach a ratio that occurs less than 5 times at which point it does not matter how the ratios are allocated.

Since the fourth most common ratio occurs at most 12 times all subsequent ratios can, if necessary, be allocated to $\mathscr{T}$.

We now relabel the variables so that the suffixes $1,2, \ldots, 15$ correspond to $\mathscr{S}$ and the suffixes $16,17, \ldots, 51$ correspond to $\mathscr{T}$. The ratios in $\mathscr{T}$ can be arranged into 18 unequal pairs and renumbered so that

$$
\frac{a_{16}}{b_{16}} \neq \frac{a_{17}}{b_{17}}, \frac{a_{18}}{b_{18}} \neq \frac{a_{19}}{b_{19}}, \ldots, \frac{a_{50}}{b_{50}} \neq \frac{a_{51}}{b_{51}}
$$

Since the ratios $a_{i} / b_{i}(1 \leqq i \leqq 15)$ are not all equal the linear equations

$$
\begin{aligned}
& a_{1} y_{1}+\cdots+a_{15} y_{15}=0 \\
& b_{1} y_{1}+\cdots+b_{15} y_{15}=0
\end{aligned}
$$

have a real solution with no $y_{i}$ zero. Replacing $x_{i}$ by $-x_{i}$ in (3), if necessary, and changing the signs of the corresponding coefficients we may suppose that each $y_{i}>0$.

Let $P$ be large and positive. For $1 \leqq j \leqq 15$ we choose constants $p_{j}$ and $r_{j}$ so that, taking $z_{j}=y_{j}^{1 / 5}$,

$$
0<p_{j}<z_{j}<r_{j}
$$

and $r_{j}-p_{j}$ is suitably small. Let $N(P)$ denote the number of solutions of (3) such that the variables $x_{j}$ satisfy

$$
p_{j} P<x_{j}<r_{j} P \text { for } j=1, \ldots, 15
$$

and

$$
P^{\lambda_{v}}<x_{j}<2 P^{\lambda_{v}} \text { for } j=16, \ldots, 51
$$

where $v=[(j-8) / 4]$ and $1, \lambda_{2}, \lambda_{3}, \ldots, \lambda_{10}$ are the admissible exponents determined by the process of sections 2 and 3 . 
Let

$$
\Lambda_{j}=a_{j} \alpha+b_{j} \beta \quad \text { for } j=1, \ldots, 51
$$

and

$$
T_{j}\left(\Lambda_{j}\right)=\sum e\left(\Lambda_{j} x^{5}\right)
$$

where $e(\theta)=\exp (2 \pi i \theta)$ and $x$ ranges over an interval of the form (53) or (54), depending on the suffix $j$. The number $N(P)$ of integer solutions of (3) in the box defined by (53) and (54) is given by

$$
N(P)=\int_{0}^{1} \int_{0}^{1} \prod_{j=1}^{51} T_{j}\left(\Lambda_{j}\right) d \alpha d \beta
$$

In order to evaluate this integral by the Hardy-Littlewood mathod, the equations (3) must have non-singular solutions in every $p$-adic field.

Lemma 2. If $N=51$ and no ratio $a_{i} / b_{i}$ occurs more than 25 times then the equations (3) have a non-singular solution in every $p$-adic field.

This follows from Theorems 1 and 2 of Davenport and Lewis [10].

\section{The minor arcs}

The unit square is divided up into major arcs $\mathbf{M}$, where $\alpha$ and $\beta$ both have good rational approximations, and the minor arcs $m$ which consist of the rest of the unit square. The major arc $\mathbf{M}(A, B, Q)$ consists of those $(\alpha, \beta)$ which have simultaneous rational approximations $A / Q, B / Q$ satisfying

$$
|\alpha-A / Q|<Q^{-1} P^{-4-\delta},|\beta-B / Q|<Q^{-1} P^{-4-\delta}
$$

where $(A, B, Q)=1$ and $\delta$ is a sufficiently small number, independent of $P$. The major arcs $\mathbf{M}$ are the union of those $\mathbf{M}(A, B, Q)$ for which

$$
1 \leqq Q \leqq P^{1-\delta}, 0 \leqq A, B<Q,(A, B, Q)=1 \text {. }
$$

By Dirichlet's theorem on Diophantine approximation, for any number $\Lambda$ we can find relatively prime integers $a$ and $q$ such that

$$
1 \leqq q \leqq P^{4+\delta} \text { and }|q \Lambda-a|<P^{-4-\delta} .
$$

Lemma 3. Suppose that

$$
|q \Lambda-a|<P^{-4-\delta} \text { and } P^{1-\delta}<q \leqq P^{4+\delta}
$$


where $a$ and $q$ are relatively prime integers. Then

$$
|T(\Lambda)| \ll P^{15 / 16+\delta}
$$

where the exponential sum $T$ is taken over a range of the form (53).

This version of Weyl's inequality is Lemma 15 of Davenport and Lewis [11].

Lemma 4. Suppose that

$$
|q \Lambda-a|<P^{-4-\delta} \text { and } 1 \leqq q \leqq P^{1-\delta}
$$

Let

$$
\Lambda=a / q+\gamma
$$

Then an exponential sum $T(\Lambda)$, over a range of the form (53), satisfies

$$
T(\Lambda) \ll q^{-1 / 5} \min \left(P, P^{-4}|\gamma|^{-1}\right) .
$$

This is Lemma 18 of Davenport and Lewis [11].

Lemma 5. Suppose that for some $i$ with $1 \leqq i \leqq 15$

$$
\left|T_{i}\left(\Lambda_{i}\right)\right|=P^{1-\theta}
$$

where

$$
\theta \leqq 1 / 16-2 \delta
$$

Then $\Lambda_{i}$ has a rational approximation $A / Q$ satisfying

$$
1 \leqq Q \ll P^{5 \theta},\left|\Lambda_{i}-\frac{A}{Q}\right| \ll \frac{1}{Q^{1 / 5} P^{5-\theta}}
$$

Proof. We choose a rational approximation $A / Q$ to $\Lambda_{i}$ satisfying the inequalities (60). If $Q>P^{1-\delta}$ we use Lemma 3 to obtain a contradiction to the inequality (67). Thus $Q \leqq P^{1-\delta}$ and so, from Lemma 4,

$$
P^{1-\theta}=\left|T_{i}\left(\Lambda_{i}\right)\right| \ll Q^{-1 / 5} \min \left(P, P^{-4}\left|\Lambda_{i}-\frac{A}{Q}\right|^{-1}\right)
$$

which gives the inequalities (68).

Lemma 6. Suppose that $(\alpha, \beta) \in \mathbf{m}$. Let $i$ and $j$ be two of the suffixes $1,2, \ldots, 15$ for 
which $a_{i} b_{j} \neq a_{j} b_{i}$. Then

$$
\text { either }\left|T_{i}\left(\Lambda_{i}\right)\right|<P^{15 / 16+2 \delta} \text { or }\left|T_{j}\left(\Lambda_{j}\right)\right|<P^{15 / 16+2 \delta} .
$$

Proof. Let

$$
\left|T_{i}\left(\Lambda_{i}\right)\right|=P^{1-\theta_{i}} \text { and }\left|T_{j}\left(\Lambda_{j}\right)\right|=P^{1-\theta_{j}}
$$

and suppose that (70) is false. Appealing to Lemma 5, we have rational approximations $A_{i} / Q_{i}, A_{j} / Q_{j}$ to $\Lambda_{i}$ and $\Lambda_{j}$ respectively such that

$$
\begin{aligned}
& 1 \leqq Q_{i} \ll P^{5 \theta_{i}},\left|\Lambda_{i}-\frac{A_{i}}{Q_{i}}\right| \ll Q_{i}^{-1 / 5} P^{-5+\theta_{i}} . \\
& 1 \leqq Q_{j} \ll P^{5 \theta_{j}},\left|\Lambda_{j}-\frac{A_{j}}{Q_{j}}\right| \ll Q_{j}^{-1 / 5} P^{-5+\theta_{j}}
\end{aligned}
$$

Now

$$
\left(a_{i} b_{j}-a_{j} b_{i}\right) \alpha=b_{j} \Lambda_{i}-b_{i} \Lambda_{j} \text { and }\left(a_{i} b_{j}-a_{j} b_{i}\right) \beta=a_{i} \Lambda_{j}-a_{j} \Lambda_{i}
$$

so that $\alpha$ and $\beta$ have simultaneous rational approximations $A / Q, B / Q$ with $(A, B, Q)=1$ and

$$
Q \mid\left(a_{i} b_{j}-a_{j} b_{i}\right) Q_{i} Q_{j}
$$

Thus

$$
Q \ll P^{5 \theta_{i}+5 \theta_{j}} \text {. }
$$

Further

$$
\begin{aligned}
|\alpha-A / Q| & \ll\left|\Lambda_{i}-A_{i} / Q_{i}\right|+\left|\Lambda_{j}-A_{j} / Q_{j}\right| \\
& \ll Q_{i}^{-1 / 5} P^{-5+\theta_{i}}+Q_{j}^{-1 / 5} P^{-5+\theta_{j}} \\
& \ll Q^{-1}\left(Q_{i}^{4 / 5} Q_{j} P^{-5+\theta_{i}}+Q_{i} Q_{j}^{4 / 5} P^{-5+\theta_{j}}\right) \\
& \ll Q^{-1} P^{-5+5 \theta_{i}+5 \theta_{j}}
\end{aligned}
$$

and similarly

$$
|\beta-B / Q| \ll Q^{-1} P^{-5+5 \theta_{i}+5 \theta_{j}} .
$$

If $5\left(\theta_{i}+\theta_{j}\right) \leqq 1-2 \delta$ then $(\alpha, \beta) \in \mathbf{M}(A, B, Q)$ and therefore

$$
\theta_{i}+\theta_{j}>\frac{1}{5}(1-2 \delta) \text {. }
$$


Hence

$$
\left|T_{i}\left(\Lambda_{i}\right) T_{j}\left(\Lambda_{j}\right)\right|=P^{2-\theta_{i}-\theta_{j}}<P^{9 / 5+\delta}
$$

and therefore

$$
\min \left(\left|T_{i}\left(\Lambda_{i}\right)\right|,\left|T_{j}\left(\Lambda_{j}\right)\right|\right)<P^{9 / 10+\delta}<P^{15 / 16+2 \delta} .
$$

Now let $V(\alpha, \beta)$ be the product of four sums $T_{j}\left(\Lambda_{j}\right), j \in \mathscr{S}$, with no ratio $a_{i} / b_{i}$ occurring more than twice.

Lemma 7. For any $\varepsilon>0$

$$
\int_{0}^{1} \int_{0}^{1}\left|V(\alpha, \beta) \prod_{j=16}^{51} T_{j}\left(\Lambda_{j}\right)\right| d \alpha d \beta \ll P^{2 \sigma+\varepsilon} .
$$

This follows from the definition of admissible exponents and Cauchy's inequality.

Lemma 8. For any $\varepsilon>0$

$$
\iint_{\mathrm{m}}\left|\prod_{j=1}^{51} T_{j}\left(\Lambda_{j}\right)\right| d \alpha d \beta \ll P^{21 / 2+2 \sigma+16 \delta+\varepsilon}
$$

Proof. The 15 suffixes in $\mathscr{P}$ are partitioned into blocks of equal ratios $a_{i} / b_{i}$, no block containing more than 5 suffixes. For any $(\alpha, \beta) \in \mathbf{m}$

$$
\left|T_{j}\left(\Lambda_{j}\right)\right| \ll P^{15 / 16+2 \delta}
$$

for all the suffixes in $\mathscr{S}$ apart from those in one block. For each suffix $j$ let $\mathbf{m}_{j}$ denote the subset of $\mathbf{m}$ for which

$$
\max _{i \in \mathscr{S}}\left|T_{i}\left(\Lambda_{i}\right)\right|=\left|T_{j}\left(\Lambda_{j}\right)\right|
$$

To estimate the contribution of $\mathbf{m}_{j}$ we put 4 exponential sums into a product $V=V_{j}(\alpha, \beta)$ so that at most 3 sums from the block of $a_{j} / b_{j}$ remain outside the product. The estimate (77) holds for at least 8 of the 11 sums which remain and so

$$
\begin{aligned}
\iint_{\mathrm{m}_{j}}\left|\prod_{i=1}^{51} T_{i}\left(\Lambda_{i}\right)\right| d \alpha d \beta & \ll P^{21 / 2+16 \delta} \int_{0}^{1} \int_{0}^{1}\left|V_{j}(\alpha, \beta) \prod_{i=16}^{51} T_{i}\left(\Lambda_{i}\right)\right| d \alpha d \beta \\
& \ll P^{21 / 2+2 \sigma+16 \delta+\varepsilon} .
\end{aligned}
$$

and (76) follows on summing over $j$. 


\section{The major arcs}

For $(\alpha, \beta) \in \mathbf{M}(A, B, Q)$ let

$$
\phi=\alpha-A / Q, \psi=\beta-B / Q
$$

and

$$
\frac{c_{j}}{q_{j}}=\frac{a_{j} A+b_{j} B}{Q},\left(c_{j}, q_{j}\right)=1 .
$$

Then

$$
\Lambda_{j}=a_{j} \alpha+b_{j} \beta=\frac{c_{j}}{q_{j}}+\gamma_{j}
$$

where

$$
\gamma_{j}=a_{j} \phi+b_{j} \psi
$$

Lemma 9. We have

$$
\int_{-\infty}^{\infty} \int_{-\infty}^{\infty} \prod_{j=1}^{15} \min \left(P, P^{-4}\left|\gamma_{j}\right|^{-1}\right) d \phi d \psi \ll P^{5}
$$

and for any $\tau>0$

$$
\iint_{D(\tau)} \prod_{j=1}^{15} \min \left(P, P^{-4}\left|\gamma_{j}\right|^{-1}\right) d \phi d \psi \ll P^{5-6 \tau}
$$

where

$$
D(\tau)=\left\{(\phi, \psi):|\phi|>P^{-5+\tau},|\psi|>P^{-5+\tau}\right\} .
$$

This may be proved in the same way as Lemma 22 of Davenport and Lewis [11], since 14 of the ratios $a_{i} / b_{i}$ can be arranged into 7 unequal pairs.

Lemma 10. For any $\varepsilon>0$ and $\lambda=\lambda_{2}=0.806779 \ldots$ we have

$$
\sum_{A, B}\left(q_{1} \ldots q_{15}\right)^{-1 / 5}\left(q_{16} \ldots q_{19}\right)^{-\lambda / 16} \ll Q^{-1-\lambda / 8+\varepsilon} \ll Q^{-11 / 10}
$$

where the summation is over

$$
0 \leqq A, B<Q,(A, B, Q)=1
$$

and $q_{j}$ is defined by (81). 
This may be proved in the same way as Lemma 35 of Davenport and Lewis [9] since, in their notation, we have

$$
v \geqq 3, \theta_{i} \leqq 1+\lambda / 8 \text { and } \theta_{1}+\cdots+\theta_{v}=3+\lambda / 4
$$

Their arguments then show that the sum in (87) is

$$
\ll Q^{-1-\lambda / 4} \sum_{u_{1} \ldots u_{v} \mid K Q}\left(u_{1} \ldots u_{v}\right)^{\lambda / 8}
$$

where $K$ is fixed and non-zero, which implies (87).

Our next step is to take an arbitrary small positive number $\omega$ and to estimate the contribution of those major arcs with $Q>P^{\omega}$.

Lemma 11. The contribution made to the integral by all those major arcs $\mathbf{M}(A, B, Q)$ with $Q>P^{\omega}$ is (provided $\delta<\omega / 80$ )

$$
\ll P^{1+4 \sigma-(1 / 20) \omega} \text {. }
$$

This may be proved in the same way as Lemma 37 of Davenport and Lewis [9], using Lemmas 9 and 10 in place of their Lemmas 35 and 36.

The next step is to replace the remaining major arcs $\mathbf{M}(A, B, Q)$ by truncated major arcs $M_{0}(A, B, Q)$ defined by

$$
|\alpha-A / Q|<P^{-5+\tau},|\beta-B / Q|<P^{-5+\tau}
$$

where $\tau$ is a small positive constant.

Lemma 12. The total difference between the contributions of the major arcs $\mathbf{M}(A, B, Q)$ and the truncated major arcs $\mathbf{M}_{0}(A, B, Q)$ with $Q \leqq P^{\omega}$ is

$$
\ll P^{1+4 \sigma-6 \tau} \text {. }
$$

This may be proved in the same way as Lemma 25 of Davenport and Lewis [11], with our Lemma 9 replacing their Lemma 22.

Thus

$$
N(P)=\sum_{Q \leqq P^{\infty}} \sum_{A, B} \int_{\mathbf{M}_{0}} \int_{A, B, Q)} \prod_{j=1}^{51} T_{j}\left(\Lambda_{j}\right) d \alpha d \beta+o\left(P^{1+4 \sigma}\right) .
$$

\section{Proof of Theorem 1}

On the truncated major arcs $\mathbf{M}_{0}(A, B, Q)$ there is a good approximation to the exponential sums $T_{j}\left(\Lambda_{j}\right)$. 
Lemma 13. Let $(\alpha, \beta) \in \mathbf{M}_{0}(A, B, Q)$. For $1 \leqq j \leqq 15$

$$
T_{j}\left(\Lambda_{j}\right)=q_{j}^{-1} S\left(c_{j}, q_{j}\right) I_{j}\left(\gamma_{j}\right)+O\left(P^{2 \eta}\right)
$$

where

$$
S(c, q)=\sum_{x=1}^{q} e_{q}\left(c x^{5}\right)
$$

and

$$
I_{j}\left(\gamma_{j}\right)=\int_{p_{j} P}^{r_{j} P} e\left(\gamma_{j} x^{5}\right) d x
$$

and $\eta$ is small if $\omega$ and $\tau$ are small. If $16 \leqq j \leqq 51$ then

$$
T_{j}\left(\gamma_{j}\right)=q_{j}^{-1} S\left(c_{j}, q_{j}\right) P^{\lambda_{v}}+O\left(P^{2 \eta \lambda_{v}}\right)
$$

where $v=[(j-8) / 4]$.

This is essentially Lemma 27 of Davenport and Lewis [11].

Substituting these approximations into (92) we obtain, as in [11, Lemma 28]

$$
N(P)=S\left(P^{\omega}\right) I\left(P^{-5+\tau}\right)+o\left(P^{1+4 \sigma}\right)
$$

where

$$
S\left(P^{\omega}\right)=\sum_{Q \leqq P^{\omega}} \sum_{A, B} \prod_{j=1}^{S 1} q_{j}^{-1} S\left(c_{j}, q_{j}\right)
$$

and

$$
I\left(P^{-5+\tau}\right)=C P^{4(\sigma-1)} \iint \prod_{j=1}^{15} I_{j}\left(\gamma_{j}\right) d \phi d \psi
$$

where $C$ is a positive constant and the integration is over the region

$$
|\phi|<P^{-5+\tau},|\psi|<P^{-5+\tau}
$$

Lemma 14. If the equations (3) have a non-singular p-adic solution for every prime $p$ then

$$
S\left(P^{\infty}\right)=S+o(1) \quad \text { as } \quad P \rightarrow \infty
$$

where $S$ is a positive constant. 
This is essentially a combination of Lemmas 29 and 31 of Davenport and Lewis [11].

Lemma 15. We have

$$
I\left(P^{-5+\tau}\right)=C_{0} P^{1+4 \sigma}(1+o(1)) \quad \text { as } \quad P \rightarrow \infty
$$

where $C_{0}$ is positive and independent of $P$.

This is essentially Lemma 30 of Davenport and Lewis [11], we have $C_{0}>0$ since the box defined by (53) contains a non-singular real solution of the equations (23).

Thus, if no ratio $a_{i} / b_{i}$ occurs more than 21 times

$$
N(P)=C_{0} S P^{1+4 \sigma}(1+o(1))>0
$$

which completes the proof of Theorem 1.

\section{Proof of Theorem 2}

A single additive equation of odd degree $k$

$$
c_{1} x_{1}^{k}+\cdots+c_{N} x_{N}^{k}=0
$$

has a non-trivial solution in integers provided that

$$
N \geqq \max \left(G^{*}(k), \Gamma^{*}(k)\right)
$$

where $G^{*}(k)$ is the number of variables needed for the Hardy-Littlewood method to work and $\Gamma^{*}(k)$ is the number of variables needed to ensure that (103) has non-trivial $p$-adic solutions for every prime $p$.

A. Tietäväinen [12] has shown that as $k \rightarrow \infty$ through odd values

$$
\varlimsup_{k \rightarrow \infty, k \text { odd }} \frac{\Gamma^{*}(k)}{k \log k}=\frac{1}{\log 2}
$$

Davenport and Lewis [8] showed that for any $\delta>0$ and sufficiently large $k>k_{0}(\delta)$

$$
G^{*}(k)<(4+\delta) k \log k
$$

and the methods of Vaughan [13], adapted from those of I. M. Vinogradov [16], show that

$$
G^{*}(k)<(3+\delta) k \log k
$$

and so the equation (103) has a non-trivial solution in integers provided that

$$
N>(3+\delta) k \log k
$$


Vaughan has also remarked [13, 15 p. 131] that another method of Vinogradov [17] can be adapted to show that

$$
G^{*}(k)<(2+\delta) k \log k
$$

which would imply the case $R=1$ of Theorem 2 . However, since there is no detailed proof of (109) in print we shall use only the weaker result (108), which is adequate for our purposes.

Lemma 16. Let $\varepsilon>0$ and let $k>k_{0}(\varepsilon, R)$ be a sufficiently large odd integer. If $N$ satisfies (4) then the equations (1) have a non-trivial p-adic solution for every prime $p$.

This was proved in [6]; however, for the analytic arguments we need non-singular $p$-adic solutions.

Lemma 17. Let $\varepsilon>0$ and put

$$
N_{0}(R)=\frac{(2+\varepsilon)}{\log 2} R^{2} k \log k
$$

For $S=1, \ldots, R$ let $Q_{s}$ denote the minimum number of terms that occur, with at least one non-zero coefficient, in any $S$ independent linear combinations of $f_{1}, \ldots, f_{R}$. Suppose that $k>k_{0}(\varepsilon, R)$ is a sufficiently large odd integer and that

$$
Q_{s} \geqq N_{0}(S) \text { for } S=1, \ldots, R \text {. }
$$

Then for every prime $p$ the equations (1) have a non-singular p-adic solution.

This may be deduced from Lemma 16 using the same argument as Theorem 4 of Davenport and Lewis [11].

We deduce Theorem 2 from Lemma 17 by an application of the Hardy-Littlewood method along the same lines as Davenport and Lewis [11], which shows that

$$
(4+\delta) R k \log k
$$

variables are sufficient for the analytic arguments (provided that the coefficients are in general position). It may be possible to replace 4 by 3 or even by 2 , along the lines of (108) and (109), however, as

$$
4 R k \log k<\frac{2}{\log 2} R^{2} k \log k
$$

for $R \geqq 2$ such an improvement in the analytic method would give no new information unless it was matched by an improvement in the $p$-adic results. Since this proof closely follows that of Davenport and Lewis [11] only a brief outline is given. 
We suppose that the $N$ variables contain $2 \ell+3 k$ sets of cardinality $R$ where for each set of $R$ variables the corresponding submatrix of $\left(a_{i j}\right)$ is non-singular and $\ell$ is the least integer such that

$$
R k(1-1 / k)^{\ell}<\frac{3}{2 k(2 \log k+\log \log k+3)}
$$

Then, for fixed $R$,

$$
\ell \sim 2 k \log k \quad \text { as } \quad k \rightarrow \infty \text {. }
$$

Let $\rho^{-1}=2 k^{2}(2 \log k+\log \log k+3)$ and define the major arc $\mathbf{M}(\mathbf{A}, Q)$ to consist of all those $\alpha=\left(\alpha_{1}, \ldots, \alpha_{R}\right)$ satisfying

$$
\left|\alpha_{i}-A_{i} / Q\right|<Q^{-1} P^{-k+k \rho}, i=1, \ldots, R,
$$

for integers $Q, A_{1}, \ldots, A_{R}$ satisfying

$$
1 \leqq Q<P^{k \rho}, 0 \leqq A_{i}<Q,\left(Q, A_{1}, \ldots, A_{R}\right)=1 .
$$

The minor arcs $\mathbf{m}$ consist of the rest of the $R$-dimensional unit cube.

Lemma 18. Let $\Lambda_{1}, \ldots, \Lambda_{R}$ be linearly independent forms in $\alpha_{1}, \ldots, \alpha_{R}$ with integral coefficients. Let

$$
T_{j}\left(\Lambda_{j}\right)=\sum e\left(\Lambda_{j} x^{k}\right)
$$

where the summation is over a range of length $O(P)$. If $\alpha \in \mathbf{m}$ then

$$
\prod_{j=1}^{R}\left|T_{j}\left(\Lambda_{j}\right)\right| \ll P^{R-\rho}
$$

This is essentially Lemma 19 of Davenport and Lewis [11] and we see that the $3 k$ sets of $R$ independent variables save $P^{u}$ where $u$ is the term on the right in (113). The $2 \ell$ sets of $R$ independent variables are used with the technique of diminishing ranges.

Let $\theta=(1-1 / k)$ and

$$
P_{v}=P^{\theta^{v-1}} \text { for } v=1, \ldots, \ell
$$

The $2 \ell$ sets of $R$ variables $B_{1}, \ldots, B_{\ell}, B_{1}^{\prime}, \ldots, B_{\ell}^{\prime}$ are allowed to range over values

$$
p_{j} P_{v}<x_{j}<r_{j} P_{v}, x_{j} \in B_{v} \text { or } B_{v}^{\prime}
$$

where $0<p_{j}<r_{j}$. Let

$$
U_{j}\left(\Lambda_{j}\right)=\sum e\left(\Lambda_{j} x_{j}^{k}\right)
$$

where $x_{j}$ ranges over an interval of the form (120). 
Lemma 19. We have

$$
\int_{0}^{1} \ldots \int_{0}^{1} \prod_{j \in \mathscr{B}}\left|U_{j}\left(\Lambda_{j}\right)\right| d \alpha \ll\left(P_{1} \ldots P_{\ell}\right)^{R}
$$

where $\mathscr{B}$ is the set of $2 \ell R$ variables.

This is Lemma 14 of Davenport and Lewis [11], it saves $P^{v}$ where

$$
v=R k\left(1-\theta^{\ell}\right)
$$

Since $u>R k \theta^{\ell}$ we have saved an exponent greater than $R k$ on the minor arcs and the $3 k$ sets of $R$ variables can be used to treat the major arcs in precisely the same way as in Davenport and Lewis [11], provided that the equations have non-singular $p$-adic solutions. Thus it is now sufficient to prove that we may assume that

(I) we can choose $2 \ell+3 k$ sets of $R$ variables, for each of which the submatrix of $\left(a_{i j}\right)$ is non-singular; and

(II) $Q_{s} \geqq N_{0}(S)$ for $S=1, \ldots, R$.

Lemma 20. Suppose that any linear combination of $f_{1}, \ldots, f_{R}$ contains more than $(n-1) R$ variables with coefficients not all zero. Then it is possible to select $n$ disjoint $R \times R$ submatrices, each of rank $R$, from the coefficient matrix $\left(a_{i j}\right)$.

This is essentially Lemma 12 of Davenport and Lewis [11], they proved it with a particular value for $n$ but the general case may be proved in exactly the same way. We can now replace condition (I) by

$$
Q_{1} \geqq(2 \ell+3 k-1) R \sim 4 R k \log k \quad \text { as } \quad k \rightarrow \infty .
$$

We prove Theorem 2 by induction on $R$, the case $R=2$ was proved in [5] so we may suppose that the result has been proved for $2,3, \ldots, R-1$. If $Q_{1}$ does not satisfy (125) we can choose one form, $f_{R}$ say, to have $Q_{1}$ variables explicitly. We set these variables to zero and obtain a system of $R-1$ equations in

$$
\begin{aligned}
N-Q_{1} & \geqq \frac{(2+\varepsilon)}{\log 2} R^{2} k \log k-(4+\eta) R k \log k \\
> & \frac{(2+\varepsilon)}{\log 2}(R-1)^{2} k \log k
\end{aligned}
$$

variables, on taking $\eta=2 \varepsilon$ and $k>k_{0}(\varepsilon, R)$. By the inductive hypothesis, this system of equations has a non-trivial solution in integers.

Now we may suppose that (125) holds, and therefore (124) holds when $S=1$. Suppose that there is some $S$ for which

$$
Q_{s}<\frac{(2+\varepsilon)}{\log 2} S^{2} k \log k
$$


then $1<S<R$. The original $R$ equations are equivalent to a system in which $S$ of the equations contain only $Q_{s}$ variables explicitly. We set these variables to zero and obtain a system of $R-S$ equations in

$$
\begin{aligned}
N-Q_{s} & \geqq \frac{(2+\varepsilon)}{\log 2}\left(R^{2}-S^{2}\right) k \log k \\
& >\frac{(2+\varepsilon)}{\log 2}(R-S)^{2} k \log k
\end{aligned}
$$

variables. For $1 \leqq R-S<R$ these equations have a non-trivial solution in integers and this provides a solution for the equations (1).

\section{An analytical improvement}

The condition $N \geqq 51$ arises from the conditions for $p$-adic solubility in Theorem 1 . If we assume the existence of $p$-adic solutions and impose some extra conditions on the ratios $a_{i} / b_{i}$ then fewer variables are sufficient. For example, if the ratios $a_{i} / b_{i}$ are all different then the inequality

$$
2 \sigma(9)+11 / 16=10.015266>10
$$

indicates that 47 variables would be sufficient for the analytical argument. In fact 47 variables will be sufficient if we can partition the suffixes into two sets $\mathscr{S}$ and $\mathscr{T}$ such that

(i) $|\mathscr{S}|=15,|\mathscr{T}|=32$;

(ii) no ratio $a_{i} / b_{i}$ occurs more than twice in $\mathscr{S}$; and

(iii) no ratio $a_{i} / b_{i}$ occurs more than 16 times in $\mathrm{T}$.

\section{REFERENCES}

1. S. Chowla and G. Shimura, On the representation of zero by a linear combination of $k$ th powers, Norske Vid. Selsk. Forh. 36 (1963), 169-178.

2. R. J. CooK, Simultaneous quadratic equations, J. London Math. Soc. 4 (1971), 319-326.

3. R. J. Cook, A note on a lemma of Hua, Quart. J. Math. (Oxford) 23 (1972), 287-288.

4. R. J. Cook, Pairs of additive equations, Michigan Math. J. 19 (1972), 325-331.

5. R. J. СоoK, Pairs of additive equations II: large odd degree, J. Number Theory (to appear).

6. R. J. Cook, Simultaneous additive congruences, J. Number Theory (submitted).

7. H. Davenport, On sums of positive integral $k$ th powers, Proc. Roy. Soc. 170A (1939), 293299.

8. H. Davenport and D. J. Lewis, Homogeneous additive equations, Proc. Roy. Soc. 274A (1963), 443-460. 
9. H. Davenport and D. J. Lewis, Cubic equations of additive type, Phil. Trans. Roy. Soc. 261 A (1966), 97-136.

10. H. Davenport and D. J. Lewis, Two additive equations, Proc. Sympos. Pure Math. XII (Amer. Math. Soc., 1969), 74-98.

11. H. Davenport and D. J. Lewis, Simultaneous equations of additive type, Phil. Trans. Roy. Soc. 264A (1969), 557-595.

12. A. Tietäväınen, On a problem of Chowla and Shimura, J. Number Theory 3 (1971), 247252.

13. R. C. Vaughan, Homogeneous additive equations and Waring's problem, Acta Arith. 33 (1977), 231-253.

14. R. C. Vaughan, On pairs of additive cubic equations, Proc. London Math. Soc. 34 (1977), 354-364.

15. R. C. Vaughan, The Hardy-Littlewood Method (Cambridge University Press, 1981).

16. I. M. Vinogradov, The Method of Trigonometrical Sums in the Theory of Numbers, translated and revised by K. F. Roth and A. Davenport (Interscience, London, 1954).

17. I. M. VINOGRAdov, On an upper bound for G(n), Izvestia Akad. Nauk. SSSR 23 (1959), 637 642.

UNIVERSITY OF SHEFFIELD

SHEFFIELD 10. 